\title{
Relationship between Bondi-Sachs quantities and source of gravitational radiation in asymptotically de Sitter spacetime
}

\author{
Xiaokai He, ${ }^{1,2}$, 田 Jiliang Jing,, , 团 and Zhoujian $\mathrm{Cao}^{3,4}$,团 \\ ${ }^{1}$ Department of Physics, Key Laboratory of Low Dimensional \\ Quantum Structures and Quantum Control of Ministry of Education, \\ and Synergetic Innovation Center for Quantum Effects and Applications, \\ Hunan Normal University, Changsha, Hunan 410081, P. R. China \\ ${ }^{2}$ School of Mathematics and Computational Science, \\ Hunan First Normal University, Changsha 410205, China \\ ${ }^{3}$ Department of Astronomy, Beijing Normal University, Beijing 100875, China \\ ${ }^{4}$ Academy of Mathematics and Systems Science, Chinese Academy of Sciences, Beijing 100190, China
}

\begin{abstract}
Gravitational radiation plays an important role in astrophysics. Based on the fact that our universe is expanding, the gravitational radiation when a positive cosmological constant is presented has been studied along with two different ways recently, one is the Bondi-Sachs (BS) framework in which the result is shown by BS quantities in the asymptotic null structure, the other is the perturbation approach in which the result is presented by the quadrupoles of source. Therefore, it is worth to interpret the quantities in asymptotic null structure in terms of the information of the source. In this paper, we investigate this problem and find the explicit expressions of BS quantities in terms of the quadrupoles of source in asymptotically de Sitter spacetime. We also estimate how far away the source is, the cosmological constant may affect the detection of the gravitational wave.
\end{abstract}

PACS numbers: 95.30.Qd, 52.27.Ny, 04.70.-s

\section{INTRODUCTION}

Almost one hundred years ago, Einstein provided a relativistic description of gravitational wave by linearizing the field equation of general relativity (GR). Moreover he calculated the energy carried away by gravitational radiations emitted by a varying mass quadruple moment in harmonic gauge condition [1]. However, due to the complexity of the diffeomorphism of GR, even the existence of gravitational waves in GR caused a great controversy for a long time [2]. This controversy was finally resolved theoretically in 1960s by the work of Bondi, Sachs and others [3, 4] ]. In their work, they constructed Bondi-Sachs (BS) framework and obtained the mass loss formula in terms of the so-called "news function".

It should be noted that Einstein's calculations, as well as the BS framework in GR, used the field equations without cosmological constant. In the past years, there are strong evidences [5-10] from many independent astronomical observations show that our present universe is in a state of accelerating expansion and is best modeled by cold dark matter with a positive cosmological constant $\Lambda$. Then it is worth to recheck the behavior of gravitational wave based on the Einstein's equation with a cosmological constant

$$
R_{\mu \nu}-\frac{1}{2} R g_{\mu \nu}+\Lambda g_{\mu \nu}=8 \pi T_{\mu \nu}
$$

where we have used geometric units with $c=G=1$.

Very recently, three gravitational wave detection events have been confirmed [11 13]. The sources of these two events locate about billion light years away which is not very far away compared to the cosmological scale. The experiment results show that the theory of Einstein, Bondi and others [1, 3, 4] works quite well. No sign of cosmological effect on gravitational wave shows up. In the near future when the sensitivity of advanced LIGO and other ground based detectors grows more, farther gravitational wave events will be detected and the effect of cosmological constant will become more and more significant. Surprisingly, Ashtekar and his coworkers showed that the cosmological constant $\Lambda$ may change the behavior of gravitational wave strongly no matter how small it is [14]. They found theoretically that gravitational wave does not exist for such far away sources if the spacetime is strongly asymptotical de Sitter [14 16].

\footnotetext{
*Xiaokai He: hexiaokai77@163.com

† Jiliang Jing: jljing@hunn.edu.cn

¥Zhoujian Cao: zjcao@amt.ac.cn
} 
Later, they used perturbation method based on the work of de Vega [17] to find that the gravitational wave may exist also and gave the quadrupole formula when the cosmological constant presents. In Refs. [18, 19], we analyzed the asymptotic behavior of spacetime with a cosmological constant by the BS framework. By proposing a new Bondi-type out going boundary condition for Einstein equation, we reconcile the theoretical tension between the cosmological constant and the existence of gravitational wave. More explicitly, we calculated the Newman-Penrose scalar $\Psi_{4}$ which reduce to Bondi's original result when $\Lambda$ goes to zero and found that when gravitational radiation was emitted from the isolated source, the conformal boundary of spacetime with cosmological constant is not conformally flat, i.e., the spacetime is only weakly asymptotically de Sitter. In Ref. [20], Saw discussed our new boundary condition based on Newman-Penrose formalism. Recently, Bishop use BS framework to investigate the solution of linearized Einstein equations with a cosmological constant and showed that gravitational wave energy conservation does not normally apply to inertial observers [21].

It is worth to interpret the asymptotic null structure in terms of the information of the source. For the case of Einstein equation without cosmological constant, Coleman investigated the relations between harmonic and BS coordinates by solving wave equations in 1974 and found an approximation to the news function in terms of the energy momentum tensor of the source 22. But for the case of Einstein equation with a cosmological constant, there is little results yet about the relationship between the gravitational wave "news" in BS framework and the source behavior. In this work, we intend to investigate this problem based on the result of Ref. [23] and discuss the outgoing boundary condition in the presence of a cosmological constant. We found that Coleman's method is hard to be generalized to the case including a cosmological constant. Different to Coleman's method which transforms the BS coordinate to harmonic coordinate, we transform instead the harmonic coordinate to BS coordinate and finally express quantities appeared in our new outgoing boundary condition and gravitational wave "news" in terms of the quadrupole of the source.

The rest of the paper is organized as follows. In the next section, we will solve the linearized Einstein equation with a cosmological constant for bounded gravitational wave sources. In Sec. III, we transform the solution got in Sec. II to the transverse-traceless gauge. After that we find out the Bondi-Sachs coordinate based on the transverse-traceless gauge in Sec. IV. Based on the new Bondi-type out going boundary condition, in Sec. V we review the result for Einstein equation together with a cosmological constant for axisymmetric spacetime and more extending our previous result to general spacetime case. Along the description in Sec. V, we give the expression of BS quantities in terms of the quadrupole of the gravitational wave source and discuss our new Bondi-type out going boundary condition. In section VI, we calculate the gravitational news function for an equal mass binary system based on the result obtained in Sec. V. At last, we summarize this paper in Sec. VII with some comments and discussions.

Throughout this paper, we use the signature convention $(-,+,+,+)$ for spacetime metric and geometric units with $G=c=1$. Moreover, the Einstein summation convention is also adopted. For the indexes, the Latin indices are spatial indices and run from 1 to 3, whereas Greek indices are space-time indices and run from 0 to 3 . Following the convention of Ref. [24], we use the notation ' $:=$ ' to denote the definition and the notation ' $\equiv$ ' to mean an identity.

\section{GRAVITATIONAL WAVE SOLUTION TO THE LINEARIZED EINSTEIN EQUATION WITH A COSMOLOGICAL CONSTANT}

The Minkowski spacetime is a solution to the Einstein equation without cosmological constant for empty space. And the Minkowski spacetime is a maximum symmetric spacetime. Similarly, the de Sitter spacetime is a solution to the Einstein equation with a cosmological constant for empty space. And it is also a maximum symmetric spacetime. So when the gravitational wave source is weak, it is natural to consider the perturbation of de Sitter spacetime when a positive cosmological constant is presented.

When a small perturbation of de Sitter spacetime was considered, the metric becomes [15, 23, 25]

$$
g_{\mu \nu}=\gamma_{\mu \nu}+\epsilon h_{\mu \nu}
$$

where $\gamma_{\mu \nu}$ is the de Sitter metric and $\epsilon$ is a smallness parameter. Similar to the Minkowski perturbation, it is convenient to introduce the trace-reversed perturbation

$$
\bar{h}_{\mu \nu}=h_{\mu \nu}-\frac{1}{2} \gamma_{\mu \nu} h,
$$

where $h$ is the trace of the perturbation $h \equiv \gamma^{\mu \nu} h_{\mu \nu}$. It is simpler to solve the linearized Einstein equation based on the conformal coordinate $(\eta, x, y, z)$ in which the de Sitter metric is written as

$$
d s^{2}=\frac{1}{H^{2} \eta^{2}}\left[-d \eta^{2}+d x^{2}+d y^{2}+d z^{2}\right]
$$


where $H \equiv \sqrt{\frac{\Lambda}{3}}$. By imposing more the generalized Lorentz/harmonic gauge condition

$$
\nabla^{\mu} \bar{h}_{\mu \nu}=2 H^{2} \eta \bar{h}_{0 \nu}
$$

where $\nabla^{\mu}$ is the covariant derivative operator respect to $\gamma_{\mu \nu}$, the linearized Einstein equation reduces to [23]

$$
\begin{aligned}
& \eta^{\alpha \beta} \partial_{\alpha} \partial_{\beta} \chi_{\mu \nu}+\frac{2}{\eta^{2}} \partial_{0} \chi_{\mu \nu} \\
& -\frac{2}{\eta^{2}}\left(\delta^{0}{ }_{\mu} \delta^{0}{ }_{\nu} \eta^{\alpha \beta} \chi_{\alpha \beta}+\delta^{0}{ }_{\mu} \chi_{0 \nu}+\delta^{0}{ }_{\nu} \chi_{0 \mu}\right)=-16 \pi T_{\mu \nu},
\end{aligned}
$$

where $\eta_{\alpha \beta}$ is the Minkowski metric. Here we have replaced the variable $\bar{h}_{\mu \nu}$ with

$$
\chi_{\mu \nu}:=H^{2} \eta^{2} \bar{h}_{\mu \nu}
$$

As shown in Ref. [23], in addition to the Lorenz/harmonic gauge condition (5) synchronous gauge condition can be used to make

$$
\bar{h}_{0 \mu}=0=\chi_{0 \mu}
$$

Regarding to the $\chi_{i j}$ part, based on the Hadamard ansatz, the solution to the Eq. (6) can be written as

$$
\begin{aligned}
\chi_{i j}(\eta, \vec{x}) & =4 \int d^{3} \vec{x} \frac{1}{\left|\vec{x}-\vec{x}^{\prime}\right|} T_{i j}\left(\eta-\left|\vec{x}-\vec{x}^{\prime}\right|, \vec{x}^{\prime}\right) \\
& +4 \int d^{3} \vec{x}^{\prime} \int_{-\infty}^{\eta-\left|\vec{x}-\vec{x}^{\prime}\right|} \frac{d \eta^{\prime}}{\eta^{\prime}} \frac{\partial T_{i j}\left(\eta^{\prime}, \vec{x}^{\prime}\right)}{\partial \eta^{\prime}}
\end{aligned}
$$

where $\vec{x} \equiv(x, y, z)$ and $|\vec{x}| \equiv \sqrt{x^{2}+y^{2}+z^{2}}$. The integration is respect to the gravitational wave source region which is denoted with coordinate $\vec{x}^{\prime}$. In the wave zone where $|\vec{x}|>>\left|\vec{x}^{\prime}\right|$, we can use the approximation $\left|\vec{x}-\vec{x}^{\prime}\right| \simeq R:=|\vec{x}|$. Then we can get

$$
\begin{aligned}
\chi_{i j}(\eta, \vec{x})= & \frac{4 \eta}{r(\eta-R)} \int d^{3} \vec{x}^{\prime} T_{i j}\left(\eta-R, \vec{x}^{\prime}\right) \\
& -4 \lim _{\eta^{\prime} \rightarrow-\infty} \frac{1}{\eta^{\prime}} \int d^{3} \vec{x}^{\prime} T_{i j}\left(\eta^{\prime}, \vec{x}^{\prime}\right) \\
& +4 \int d^{3} x^{\prime} \int_{-\infty}^{\eta-R} d \eta^{\prime} \frac{T_{i j}\left(\eta^{\prime}, x^{\prime}\right)}{\eta^{\prime 2}}
\end{aligned}
$$

via a partial integration.

In order to relate the above solution to the BS framework, it is simpler to express the solution in the cosmological coordinate $(t, x, y, z)$ in which the de Sitter metric can be written as

$$
d s^{2}=-d t^{2}+e^{2 H t}\left[d x^{2}+d y^{2}+d z^{2}\right]
$$

The coordinate $x, y, z$ are the same to the ones in the above conformal coordinate while the coordinate $t$ is related to the above $\eta$ through

$$
\eta=-\frac{1}{H} e^{-H t}
$$

Based on the cosmological coordinate, by using the matter conservation equations, the solution (10) can be expressed as

$$
\begin{aligned}
\chi_{i j}= & \frac{2}{R}\left(e^{-H t}+H R\right)\left[\ddot{Q}_{i j}-2 H \dot{Q}_{i j}+H \dot{\bar{Q}}_{i j}\right] \\
& -2 H\left[\ddot{Q}_{i j}-3 H \dot{Q}_{i j}+H \dot{\bar{Q}}_{i j}+2 H^{2} Q_{i j}-H^{2} \bar{Q}_{i j}\right],
\end{aligned}
$$


where $R \equiv \sqrt{x^{2}+y^{2}+z^{2}}$. Here we have used the notation $Q_{i j}$ for the mass quadrupole moment and $\bar{Q}_{i j}$ for the pressure quadrupole moment

$$
\begin{aligned}
Q^{i j} & =\int_{\text {source }} d^{3} \vec{x} \sqrt{p} \rho x^{i} x^{j}, \\
\bar{Q}^{i j} & =\int_{\text {source }} d^{3} \vec{x} \sqrt{p} \pi x^{i} x^{j},
\end{aligned}
$$

where $p$ is the determinant of the three metric $p_{\mu \nu}$ respect to the constant $t$ slice, $\rho \equiv T_{\mu \nu} n^{\mu} n^{\nu}$ with $n^{\mu}$ the future directed normal vector respect to the constant $t$ slice, and $\pi \equiv T_{\mu \nu} p^{\mu \nu}$. For lower index $Q_{i j}$ and $\bar{Q}_{i j}$ we used $p_{i j}$ to lower the indexes.

It is easy to see that the solution (13) recovers the solution to the Einstein equation without cosmological constant [1] as the cosmological constant goes to zero.

\section{REEXPRESS THE SOLUTION TO LINEARIZED EINSTEIN EQUATION WITH A COSMOLOGICAL CONSTANT USING TRANSVERSE TRACELESS GAUGE}

According to the discussion in the above section, the solution to the linearized Einstein equation around de Sitter spacetime can be written as

$$
d s^{2}=-d t^{2}+e^{2 H t}\left(\delta_{i j}+\epsilon \chi_{i j}\right) d x^{i} d x^{j}
$$

where $\chi_{i j}$ is given by Eq. (13).

In order to avoid the caustics of null curves, we consider Bondi-Sachs framework within the spacetime region out of some cylinder. So it is convenient to consider a spherical coordinate $(R, \theta, \phi)$ respect to the Cartesian coordinate $(x, y, z)$ above

$$
\begin{aligned}
& x=R \sin \theta \cos \phi, \\
& y=R \sin \theta \sin \phi, \\
& z=R \cos \theta .
\end{aligned}
$$

Then the metric (16) can be described in $(t, R, \theta, \phi)$ as

$$
\begin{aligned}
d s^{2}= & -d t^{2}+e^{2 H t}\left[\left(1+\epsilon \chi_{\hat{R} \hat{R}}\right) d R^{2}+2 \epsilon R \chi_{\hat{R} \hat{\theta}} d R d \theta\right. \\
& +2 \epsilon R \sin \theta \chi_{\hat{R} \hat{\phi}} d R d \phi+R^{2}\left(1+\epsilon \chi_{\hat{\theta} \hat{\theta}}\right) d \theta^{2} \\
& \left.+2 \epsilon R^{2} \sin \theta \chi_{\hat{\theta} \hat{\phi}} d \theta d \phi+R^{2} \sin ^{2} \theta\left(1+\epsilon \chi_{\hat{\phi} \hat{\phi}}\right) d \phi^{2}\right]
\end{aligned}
$$


where

$$
\begin{aligned}
\chi_{\hat{R} \hat{R}}= & \chi_{33} \cos ^{2} \theta+\chi_{11} \cos ^{2} \phi \sin ^{2} \theta+\chi_{13} \cos \phi \sin 2 \theta \\
& +\chi_{23} \sin 2 \theta \sin \phi+\chi_{22} \sin ^{2} \theta \sin ^{2} \phi \\
& +\chi_{12} \sin ^{2} \theta \sin 2 \phi, \\
\chi_{\hat{R} \hat{\theta}}= & \left(\chi_{13} \cos \phi+\chi_{23} \sin \phi\right) \cos ^{2} \theta \\
& -\left(\chi_{13} \cos \phi+\chi_{23} \sin \phi\right) \sin ^{2} \theta \\
& +\left(-\chi_{33}+\chi_{11} \cos ^{2} \phi+\chi_{22} \sin ^{2} \phi\right. \\
& \left.+\chi_{12} \sin 2 \phi\right) \cos \theta \sin \theta, \\
\chi_{\hat{R} \hat{\phi}}= & \left(\chi_{23} \cos \phi-\chi_{13} \sin \phi\right) \cos \theta \\
& +\frac{1}{2} \sin \theta\left(2 \chi_{12} \cos 2 \phi+\chi_{22} \sin 2 \phi-\chi_{11} \sin 2 \phi\right), \\
\chi_{\hat{\theta} \hat{\theta}}= & \chi_{33} \sin { }^{2} \theta-\sin 2 \theta\left(\chi_{13} \cos \phi+\chi_{23} \sin \phi\right) \\
& +\cos ^{2} \theta\left(\chi_{11} \cos { }^{2} \phi+\chi_{22} \sin ^{2} \phi+\chi_{12} \sin 2 \phi\right), \\
\chi_{\hat{\theta} \hat{\phi}}= & \sin \theta\left(-\chi_{23} \cos \phi+\chi_{13} \sin \phi\right) \\
& +\frac{1}{2} \cos \theta\left(2 \chi_{12} \cos 2 \phi+\left(-\chi_{11}+\chi_{22}\right) \sin 2 \phi\right), \\
\chi_{\hat{\phi} \hat{\phi}}= & \chi_{22} \cos ^{2} \phi-2 \chi_{12} \cos \phi \sin \phi+\chi_{11} \sin ^{2} \phi .
\end{aligned}
$$

It is well known that the gravitational waves have only two physical freedom in Minkowski background. In 1999, Vega has shown that the gravitational waves in de Siiter background also share this property [17, 26]. When we consider a distant observer in the wave zone measures gravitational waves from a radiating source at origin, the metric form will be simple in transverse traceless (TT) gauge. For this purpose, we need to project out the non-TT part of the perturbation, and then the metric can be written as

$$
\begin{aligned}
d s^{2}= & -d t^{2}+e^{2 H t}\left[d R^{2}+R^{2}\left(1+\epsilon h_{+}\right) d \theta^{2}\right. \\
& \left.+R^{2} \sin ^{2} \theta\left(1-\epsilon h_{+}\right) d \phi^{2}+2 \epsilon R^{2} \sin \theta h_{\times} d \theta d \phi\right],
\end{aligned}
$$

with

$$
\begin{aligned}
& h_{+}=\frac{1}{2}\left(\chi_{\hat{\theta} \hat{\theta}}-\chi_{\hat{\phi} \hat{\phi}}\right), \\
& h_{\times}=\chi_{\hat{\theta} \hat{\phi}} .
\end{aligned}
$$

To see this, we can use the projection operator

$$
P_{i}{ }^{j}=\delta_{i}{ }^{j}-n_{i} n^{j},
$$

with $n^{i}=\left(\frac{\partial}{\partial R}\right)^{i}$ which is normal respect to the Euclidian metric $\delta_{i j}$ and raising and lowering index with metric $\delta_{i j}$. Then the transverse-traceless part of the perturbation can be calculated as [27]

$$
\begin{aligned}
h_{i j}^{T T}= & \left(P_{i}^{k} P_{j}^{l}-\frac{1}{2} P_{i j} P^{k l}\right) h_{k l} \\
= & \left(\delta_{i}^{k} \delta_{j}^{l}-\delta_{i}^{k} n_{j} n^{l}-n_{i} n^{k} \delta_{j}^{l}\right. \\
& \left.+\frac{1}{2} n_{i} n_{j} n^{k} n^{l}+\frac{1}{2} \delta_{i j} n^{k} n^{l}\right) h_{k l}
\end{aligned}
$$

Straight forward calculation gives

$$
\begin{gathered}
\chi_{\hat{R} \hat{R}}^{T T}=0, \chi_{\hat{R} \hat{\theta}}^{T T}=0, \chi_{\hat{R} \hat{\phi}}^{T T}=0, \\
h_{+}=\chi_{\hat{\theta} \hat{\theta}}^{T T}=-\chi_{\hat{\phi} \hat{\phi}}^{T T}=\frac{1}{2}\left(\chi_{\hat{\theta} \hat{\theta}}-\chi_{\hat{\phi} \hat{\phi}}\right), \\
h_{\times}=\chi_{\hat{\theta} \hat{\phi}}^{T T}=\chi_{\hat{\theta} \hat{\phi}} .
\end{gathered}
$$




\section{REEXPRESS SOLUTION TO LINEARIZED EINSTEIN EQUATION WITH A COSMOLOGICAL CONSTANT IN BONDI-SACHS FRAMEWORK}

We look for the corresponding Bondi-Sachs coordinate $(u, r, \theta, \phi)$ for the metric (19). In order to find the coordinate $u$ we have to solve the Eikonal equation

$$
g^{a b} \nabla_{a} u \nabla_{b} u=0
$$

We solve this equation approximately with the form

$$
u=-\frac{1}{H} \ln \left(H R+e^{-t H}\right)+\epsilon f_{1}(t, R, \theta, \phi)+o(\epsilon) .
$$

Here the leading order term corresponds to the $u$ coordinate for de Sitter space. Plug the metric (19) into the equation (24), we get

$$
e^{-t H} \frac{\partial f_{1}}{\partial R}+\frac{\partial f_{1}}{\partial t}=0
$$

Since one special Bondi-Sachs coordinate is enough for our usage, we need only find one special solution to the above equation. For simplicity, we chose the special solution $f_{1}=0$.

Now we can assume the coordinate transformation from $(t, R, \theta, \phi)$ to $(u, r, \theta, \phi)$ reads as 21.

$$
\begin{aligned}
& u=-\frac{1}{H} \ln \left(H R+e^{-t H}\right)+o(\epsilon), \\
& r=e^{t H} R+\epsilon f_{2}(t, R, \theta, \phi)+o(\epsilon) .
\end{aligned}
$$

Once again, the leading order term in the above $r$ equation corresponds to the $r$ coordinate for de Sitter space. Because the $r$ should be a luminosity distance parameter, within $(u, r, \theta, \phi)$ coordinate we have

$$
g_{22} g_{33}-g_{23}^{2}=r^{4} \sin ^{2} \theta,
$$

where $g_{22}, g_{23}$ and $g_{33}$ are the metric coefficients within $(u, r, \theta, \phi)$ coordinate. Direct calculation shows

$$
f_{2}(t, R, \theta, \phi)=0 .
$$

So based on the above coordinate transformation, we can calculate the metric coefficients within Bondi-Sachs coordinate $(u, r, \theta, \phi)$ as

$$
\begin{aligned}
g^{\theta \theta} & =\frac{1}{r^{2}}-\frac{\epsilon h_{+}}{r^{2}}, \\
g^{\theta \phi} & =-\frac{\epsilon h_{\times}}{r^{2} \sin \theta}, \\
g^{\phi \phi} & =\frac{1}{r^{2} \sin ^{2} \theta}+\frac{\epsilon h_{+}}{r^{2} \sin ^{2} \theta} .
\end{aligned}
$$

Recalling the result (17), and using the coordinate relation between $(t, R, \theta, \phi)$ and $(u, r, \theta, \phi)$ we have

$$
h_{+}=H^{2} A+\frac{\dot{A}}{r},
$$

with

$$
\begin{aligned}
A= & {\left[\left(\dot{Q}_{11}+H\left(\bar{Q}_{11}-2 Q_{11}\right)\right)\left(\cos ^{2} \phi \cos ^{2} \theta-\sin ^{2} \phi\right)\right.} \\
& +\left(\dot{Q}_{22}+H\left(\bar{Q}_{22}-2 Q_{22}\right)\right)\left(\sin ^{2} \phi \cos ^{2} \theta-\cos ^{2} \phi\right) \\
& +\left(\dot{Q}_{33}+H\left(\bar{Q}_{33}-2 Q_{33}\right)\right) \sin ^{2} \theta \\
& +\left(\dot{Q}_{12}+H\left(\bar{Q}_{12}-2 Q_{12}\right)\right)\left(1+\cos ^{2} \theta\right) \sin 2 \phi \\
& -\left(\dot{Q}_{13}+H\left(\bar{Q}_{13}-2 Q_{13}\right)\right) \sin 2 \theta \cos \phi \\
& \left.-\left(\dot{Q}_{23}+H\left(\bar{Q}_{23}-2 Q_{23}\right)\right) \sin 2 \theta \sin \phi\right]
\end{aligned}
$$


and

$$
h_{\times}=H^{2} B+\frac{\dot{B}}{r}
$$

with

$$
\begin{aligned}
B= & {\left[2 \sin \theta \sin \phi\left(\dot{Q}_{13}+H\left(\bar{Q}_{13}-2 Q_{13}\right)\right)\right.} \\
& -2 \sin \theta \cos \phi\left(\dot{Q}_{23}+H\left(\bar{Q}_{23}-2 Q_{23}\right)\right) \\
& +2 \cos \theta \cos 2 \phi\left(\dot{Q}_{12}+H\left(\bar{Q}_{12}-2 Q_{12}\right)\right) \\
& -\cos \theta \sin 2 \phi\left(\left(\dot{Q}_{11}-\dot{Q}_{22}\right)\right. \\
& \left.\left.+H\left(\bar{Q}_{11}-\bar{Q}_{22}-2 Q_{11}+2 Q_{22}\right)\right)\right] .
\end{aligned}
$$

When $\Lambda=0$ our results for $h_{+}$and $h_{\times}$recover the results got in the literature (say the Eqs. (9.24) and (9.25) of Ref. [27] and the Eq. (3.72) of Ref. [27]) [29]. So more explicitly we have

$$
\begin{aligned}
g^{\theta \theta} & =\frac{1}{r^{2}}-\frac{\epsilon H^{2} A}{r^{2}}-\frac{\epsilon \dot{A}}{r^{3}}, \\
g^{\theta \phi} & =-\frac{\epsilon H^{2} B}{r^{2} \sin \theta}-\frac{\epsilon \dot{B}}{r^{3} \sin \theta}, \\
g^{\phi \phi} & =\frac{1}{r^{2} \sin ^{2} \theta}+\frac{\epsilon H^{2} A}{r^{2} \sin ^{2} \theta}+\frac{\epsilon \dot{A}}{r^{3} \sin ^{2} \theta} .
\end{aligned}
$$

If the gravitational wave source is axisymmetric, the corresponding spacetime is also axisymmetric. For a axisymmetric source, the quadruple components satisfy the following relation

$$
\begin{aligned}
& Q_{22}=Q_{11}, Q_{12}=Q_{13}=Q_{23}=0 \\
& \bar{Q}_{22}=\bar{Q}_{11}, \bar{Q}_{12}=\bar{Q}_{13}=\bar{Q}_{23}=0 .
\end{aligned}
$$

These relations result in

$$
\begin{aligned}
& A=\sin ^{2} \theta\left[\dot{Q}_{33}-\dot{Q}_{11}+H\left(\bar{Q}_{33}-\bar{Q}_{11}-2\left(Q_{33}-Q_{11}\right)\right)\right] \\
& B=0 .
\end{aligned}
$$

\section{RELATED RESULT BASED ON NEW BONDI-TYPE BOUNDARY CONDITION}

\section{A. Axisymmetric case}

In Refs. [18, 19] we have proposed a new Bondi-type out going boundary condition for Einstein equation with a cosmological constant. For simplicity, we considered only axisymmetric spacetime there. Based on axisymmetric assumption, the metric can be written as following within Bondi-Sachs coordinate

$$
\begin{aligned}
d s^{2}= & -\left(V r^{-1} e^{2 \beta}-U^{2} r^{2} e^{2 \gamma}\right) d u^{2}-2 e^{2 \beta} d u d r \\
& -2 U r^{2} e^{2 \gamma} d u d \theta+r^{2}\left(e^{2 \gamma} d \theta^{2}+e^{-2 \gamma} \sin ^{2} \theta d \phi^{2}\right) .
\end{aligned}
$$

and we have shown that under the outgoing boundary condition

$$
\gamma=\Lambda f(u, \theta)+\frac{c(u, \theta)}{r}+\cdots
$$


the Einstein equation together with a cosmological constant results in

$$
\begin{aligned}
d s^{2}= & {\left[\frac{\Lambda}{3} r^{2}-\frac{c^{2} \Lambda}{6}-e^{-2 \Lambda f}\left(1+\frac{1}{2} e^{2 \Lambda f} \Lambda c^{2}+3 \Lambda \frac{\partial f}{\partial \theta}\right.\right.} \\
& \left.\left.+\Lambda \frac{\partial^{2} f}{\partial \theta^{2}}-2 \Lambda^{2}\left(\frac{\partial f}{\partial \theta}\right)^{2}\right)+\frac{2 M}{r}\right] d u^{2} \\
& -2\left(1-\frac{c^{2}}{2 r^{2}}\right) d u d r+2\left[2 c \cot \theta+\frac{\partial c}{\partial \theta}-2 \Lambda c \frac{\partial f}{\partial \theta}\right. \\
& \left.+\frac{4 c^{2} \cot \theta-U_{3} e^{2 \Lambda f}+2 c \frac{\partial c}{\partial \theta}-4 \Lambda c^{2} \frac{\partial f}{\partial \theta}}{r}\right] d u d \theta \\
& +e^{2 \Lambda f}\left[r^{2}+2 c r+2 c^{2}+\left(c^{3}+2 C\right) \frac{1}{r}\right] d \theta^{2} \\
& +e^{-2 \Lambda f}\left[r^{2}-2 c r+2 c^{2}-\left(c^{3}+2 C\right) \frac{1}{r}\right] \sin ^{2} \theta d \phi^{2},
\end{aligned}
$$

up to the order $o\left(r^{-2}\right)$. Regarding to the meaning of the notations such as $M$ and $C$, we refer readers to our previous papers [18, 19]. Based on the coordinate transformation Eqs. (27) and (28), the metric (19) seems to result in vanishing metric coefficient for $d u d \theta$. This is because the (19) solves the Einstein equation up to leading post-Newtonian order. So the back reaction such as the $d u d \theta$ term does not show up.

Equivalently, the inverse metric can be calculated as

$$
g^{\theta \theta}=e^{-2 \Lambda f}\left(\frac{1}{r^{2}}-\frac{2 c}{r^{3}}\right)=\frac{1}{r^{2}}-\frac{6 H^{2} f}{r^{2}}-\frac{2 c}{r^{3}},
$$

where the relation $\Lambda=3 H^{2}$ has been used and the higher order terms like $f^{2}, c f$ are dropped because we consider weak gravitational radiation which will be used to compare with the solution to the linearized Einstein equation got in above sections.

Comparing Eqs. (38) and (48), we can obtain

$$
f=\frac{\epsilon A}{6}, \quad c=\frac{\epsilon \dot{A}}{2}
$$

This solution results in

$$
c=3 \dot{f}
$$

which is consistent to the Eq. (19) of Ref. [18] we got previously.

When $\Lambda=0$, Eqs. (38) and (48) becomes

$$
g^{\theta \theta}=\frac{1}{r^{2}}-\frac{\epsilon \dot{A}}{r^{3}}
$$

and

$$
g^{\theta \theta}=\frac{1}{r^{2}}-\frac{2 c}{r^{3}}
$$

respectively. Combing with Eq. 430, we can get

$$
c=\frac{1}{2} \sin ^{2} \theta\left[\ddot{Q}_{33}-\ddot{Q}_{11}\right],
$$

which is exactly coincide with the result in Minkowski perturbation [27].

\section{B. General spacetime case}

In this subsection we extend our previous results to general spacetime situation. For general spacetime, the metric within Bondi-Sachs coordinate can be written as [3, 4]

$$
\begin{array}{r}
d s^{2}=-\left(V r^{-1} e^{2 \beta}-r^{2} h_{A B} U^{A} U^{B}\right) d u^{2}-2 e^{2 \beta} d u d r \\
-2 r^{2} h_{A B} U^{A} d u d x^{B}+r^{2} h_{A B} d x^{A} d x^{B},
\end{array}
$$


where the index $A$ and $B$ run through 2 and 3 which correspond to $\theta$ and $\phi$ respectively. Using the notations $U^{2}=U, U^{3}=W \csc \theta$ and

$$
h_{A B}=\left(\begin{array}{cc}
e^{2 \gamma} \cosh 2 \delta & \sinh 2 \delta \sin \theta \\
\sinh 2 \delta \sin \theta & e^{-2 \gamma} \cosh 2 \delta \sin ^{2} \theta
\end{array}\right),
$$

where $\beta, \gamma, \delta, V, U$, and $W$ are six functions of $u, r$ and points on the 2-sphere which are parameterized by $\theta$ and $\phi$. It can be easily checked that

$$
\operatorname{det}\left(h_{A B}\right)=\sin ^{2} \theta,
$$

which guarantees that $r$ is a luminosity distance parameter. When $\delta=W=0$, the line element reduces to the axisymmetric case (45).

Regarding to the boundary condition, we need to specify both $\gamma$ and $\delta$ for general spacetime. Corresponding to the new Bondi-type out going boundary condition, the specific form reads as

$$
\begin{aligned}
& \gamma=\Lambda f(u, \theta, \phi)+\frac{c(u, \theta, \phi)}{r}+\cdot \cdot \\
& \delta=\Lambda \tilde{f}(u, \theta, \phi)+\frac{\tilde{c}(u, \theta, \phi)}{r}+\cdots
\end{aligned}
$$

Based on these boundary conditions, we can solve Einstein equation together with a cosmological constant to get

$$
\begin{aligned}
g^{\theta \theta}= & \frac{e^{-2 \Lambda f} \cosh 2 \Lambda \tilde{f}}{r^{2}} \\
& +\frac{e^{-2 \Lambda f}(-2 c \cosh 2 \Lambda \tilde{f}+2 \tilde{c} \sinh 2 \Lambda \tilde{f})}{r^{3}} \\
\approx & \frac{1}{r^{2}}-\frac{6 H^{2} f}{r^{2}}-\frac{2 c}{r^{3}}, \\
g^{\theta \phi}= & -\frac{\sinh 2 \Lambda \tilde{f}}{r^{2} \sin \theta}-\frac{2 \tilde{c} \cosh 2 \Lambda \tilde{f}}{r^{3} \sin \theta} \\
\approx & -\frac{6 H^{2} \tilde{f}}{r^{2} \sin \theta}-\frac{2 \tilde{c}}{r^{3} \sin \theta} .
\end{aligned}
$$

Here " $\approx$ " means we have already dropped the nonlinear terms respect to $f, \tilde{f}, c$ and $\tilde{c}$ based on the assumption that the gravitational radiation is weak. The Einstein equation together with a cosmological constant will give us a relation

$$
c=3 \dot{f}, \quad \tilde{c}=3 \dot{\tilde{f}}
$$

Compared to (38) and (39) we have

$$
\begin{aligned}
& f=\frac{\epsilon A}{6}, \quad c=\frac{\epsilon \dot{A}}{2}, \\
& \tilde{f}=\frac{\epsilon B}{6}, \quad \tilde{c}=\frac{\epsilon \dot{B}}{2} .
\end{aligned}
$$

Explicitly, this result is consistent to the above Eqs.(61).

\section{SUMMARY AND DISCUSSION}

After the direct detection of gravitational wave including events GW150914, GW151226 and GW170104, the exciting gravitational wave astronomy era is coming. We expect to get some hints about the mysterious problems such as dark energy through gravitational wave detection. Considering the fact that our universe is expanding, we have to understand theoretically well how the cosmological constant affect the behavior of the gravitational wave.

Although the cosmological constant of our Universe is tiny, the authors in Ref. 14] theoretically found that it may change the behavior of the gravitational wave strongly. Noting that Bondi-Sachs framework is a powerful tool to treat gravitational wave problem [3, 28], we have used it to investigate the asymptotical behavior of spacetime 
[18, 19]. It was found that the original out going boundary condition proposed by Bondi and his coworkers seems break down if we consider the cosmological constant effect. Then we investigate gravitational radiation in the presence of a cosmological constant by imposed a new Bondi-type out going boundary condition which implies that the tiny cosmological constant does affect the gravitational wave perturbably. On the other hand, in Refs. [16, 23, 25], the authors solved the linearized Einstein equation together with a cosmological constant. The solutions describe the behavior of gravitational wave produced by weak sources and the quadrupole formula was given.

An interesting question is how to interpret the quantities in asymptotic null structure in BS framework in terms of the information of the source in the perturbation approach. For this purpose, it is needed to find the relation between BS coordinate used in BS framework and generalized harmonic coordinate used in linearized perturbation method. Coleman investigated this problem by solving the harmonic gauge condition in the case without a cosmological constant, but it is too complicated to solve the generalized harmonic condition in the case with a positive cosmological constant. In this paper, we transform the linearized solution to the transverse-traceless gauge firstly and then to Bondi-Sachs gauge by solving the eikonal equation and luminosity distance condition. Finally we found the explicit expression of BS quantities in terms of the quadruple (including mass quadrupole and pressure quadrupole) of the source.

By the way, it should be noted that the perturbation begins with the term of $\sqrt{\Lambda}$ quantitatively by the linearized way. While the results resulted from our new boundary condition begin with the term of $\Lambda$. Our results in this paper implies that the term of $\sqrt{\Lambda}$ is due to the gauge effect. And more, the behavior predicted by the linearized solution is exactly consistent to the results resulted from our new boundary condition. If we take the cosmological constant $\Lambda \rightarrow 0$, our result is reduced to the Coleman's one.

Now based on Eqs. (34) and (36) we can estimate how far away when a gravitational wave source is, the cosmological constant may affect the detection of the gravitational wave. If only

$$
r \gtrsim \frac{\omega}{\Lambda}
$$

where $\omega$ is the frequency of the gravitational wave, the cosmological constant may affect the detection of the gravitational wave. The observations [9] show that the amplitude of the cosmological constant $\Lambda$ is about $10^{-52} \mathrm{~m}^{-2}$ in geometric units $(c=G=1)$. If we consider LIGO type detectors, the frequency of the detected gravitational wave is about $100 \mathrm{~Hz}$ which corresponds to $10^{6} \mathrm{~m}$. So only when the source is farther than $10^{58} \mathrm{~m}\left(\approx 10^{42}\right.$ light years), the cosmological constant may affect the detection of LIGO type detectors. So we do not expect the cosmological constant may affect the detection of LIGO type detectors. Similarly, even for pulsar timing method, we do not expect the cosmological constant may affect the detection of gravitational wave. For the gravitational wave with frequency $10^{-16} \mathrm{~Hz}$ which corresponds to the age of our Universe, if the sources are $10^{40} \mathrm{~m}\left(\approx 10^{24}\right.$ light years $)$ away, the cosmological constant may affect the gravitational wave detection. But note that the time of $10^{24}$ years is much larger than the age of our Universe, we do not expect the cosmological constant may affect the gravitational wave detection done by our humanbeing. Of course, the above estimate is based on the current perturbation result. Regarding to strong gravitational wave sources, more analysis is needed.

\section{Acknowledgments}

We thank XiongJun Fang, Xiaoning Wu and Songbai Chen for many useful discussions and comments on the manuscript. Z. Cao was supported by the NSFC (No. 11375260, No. 11622546, and No. 11690023). X. He was supported by the NSFC (No. 11401199) and the Research Foundation of Education Bureau of Hunan Province, China (No. 14C0254). J. Jing was was supported by the NSFC (No. 11475061).

[1] A. Einstein, Preus. Acad. Wissen. Pt I (1918).

[2] D.Kennefick, Traveling at the speed of thought: Einstein and the quest for gravitational waves (Princeton university press, 2007).

[3] H. Bondi, M. Van der Burg, and A. Metzner, Proceedings of the Royal Society of London. Series A. Mathematical and Physical Sciences 269, 21 (1962).

[4] R. K. Sachs, Proceedings of the Royal Society of London. Series A. Mathematical and Physical Sciences 270, 103 (1962).

[5] P. de Bernardis, P. Ade, J. Bock, J. Bond, J. Borrill, A. Boscaleri, K. Coble, B. Crill, G. De Gasperis, P. Farese, et al., Nature 404, 955 (2000).

[6] E. Komatsu, K. Smith, J. Dunkley, C. Bennett, B. Gold, G. Hinshaw, N. Jarosik, D. Larson, M. Nolta, L. Page, et al., The Astrophysical Journal Supplement Series 192, 18 (2011). 
[7] S. Perlmutter, G. Aldering, M. Della Valle, S. Deustua, R. Ellis, S. Fabbro, A. Fruchter, G. Goldhaber, D. Groom, I. Hook, et al., Nature 391, 51 (1998).

[8] A. G. Riess, A. V. Filippenko, P. Challis, A. Clocchiatti, A. Diercks, P. M. Garnavich, R. L. Gilliland, C. J. Hogan, S. Jha, R. P. Kirshner, et al., The Astronomical Journal 116, 1009 (1998).

[9] P. A. Ade, N. Aghanim, C. Armitage-Caplan, M. Arnaud, M. Ashdown, F. Atrio-Barandela, J. Aumont, C. Baccigalupi, A. J. Banday, R. Barreiro, et al., Astronomy \& Astrophysics 571, A16 (2014).

[10] T. Padmanabhan, Physics Reports 380, 235 (2003).

[11] B.P.Abbott et al., Physical Review Letters 116, 061102 (2016).

[12] B.P.Abbott et al., Physical Review Letters 116, 241103 (2016).

[13] B. P. Abbott et al. (LIGO Scientific and Virgo Collaboration), Phys. Rev. Lett. 118, 221101 (2017), URL https://link.aps.org/doi/10.1103/PhysRevLett.118.221101.

[14] A. Ashtekar, B. Bonga, and A. Kesavan, Physical Review Letters 116, 051101 (2016).

[15] A. Ashtekar, B. Bonga, and A. Kesavan, Classical and Quantum Gravity 32, 025004 (2015).

[16] A. Ashtekar, B. Bonga, and A. Kesavan, Physical Review D 92, 044011 (2015).

[17] J. R. H.J. de Vega and N.Sanchez, Physical Review D 60, 044007 (1999).

[18] X. He and Z. Cao, International Journal of Modern Physics D 24, 1550081 (2015).

[19] X. He, Z. Cao, and J. Jing, International Journal of Modern Physics D 25, 1650086 (2016).

[20] V.-L. Saw, Phys. Rev. D 94, 104004 (2016), URL http://link.aps.org/doi/10.1103/PhysRevD.94.104004

[21] N. Bishop, Physical Review D 93, 044025 (2016).

[22] C. Coleman, J.Phys.A:Math,Nucl.Gen. 7, 1858 (1974).

[23] G. Date and S. J. Hoque, Phys. Rev. D 94, 064039 (2016), URL http://link.aps.org/doi/10.1103/PhysRevD.94.064039

[24] C. Liang, Introductory differential geometry and general relativity $i$, ii (2000).

[25] A. Ashtekar, B. Bonga, and A. Kesavan, Physical Review D 92, 10432 (2015).

[26] E. E. Flanagan and S. A. Hughes, New Journal of Physics 7, 204 (2005).

[27] T.W.Baumgarte and S.L.Shapiro, Numerical Relativity (Cambridge University Press, 2010).

[28] Z. Cao and X. He, Phys. Rev. D 88, 104002 (2013), URL http://link.aps.org/doi/10.1103/PhysRevD.88.104002

[29] Note that the Eqs. (9.24) and (9.25) of Ref. [27] has some typos about terms relating to $Q_{12}$ and $Q_{13}$. 